\title{
Multiple zeta functions: the double sine function and the signed double Poisson summation formula
}

\author{
Shin-ya Koyama and Nobushige Kurokawa
}

\begin{abstract}
We construct multiple zeta functions as absolute tensor products of usual zeta functions. The Euler product expression is established for the most basic case $\zeta\left(s, \mathbf{F}_{p}\right) \otimes \zeta\left(s, \mathbf{F}_{q}\right)$ by using the signed double Poisson summation formula and the theory of the double sine function.
\end{abstract}

\section{Introduction}

A zeta function is usually considered to be a meromorphic function, satisfying a functional equation, with an Euler product expression over generalized primes. There should be an associated relation between the two sets of important objects \{zeros, poles\} and \{generalized primes\}. Such a duality is usually written as an explicit formula or a trace formula.

In this paper we try to study a new kind of zeta function constructed from ordinary zeta functions using zeros and poles. We require that the zeros or poles of the new zeta function are sums of zeros or poles of the original zeta functions. We call such a new zeta function a multiple zeta function.

We are interested in the 'Euler product expression' for this new zeta function for two reasons. The first reason is the discovery itself of a new Euler product. The second reason is that, as in the case of the usual zeta functions, we would obtain some information on zeros and poles of the new zeta function via its Euler product. This might lead to a new result on zeros and poles of the original zeta functions.

The problem is non-trivial even in the simplest case, constructed from the Hasse zeta functions of two finite fields or from the Selberg zeta functions of two circles. In this paper, we use the theory of multiple sine functions to obtain a neat Euler product for this case.

In a forthcoming paper, we will establish the Euler product for the 'double Riemann zeta function', constructed from two copies of the Riemann zeta function. For that case, the Euler product can be obtained through an extension of our method based on the signed double explicit formula.

We now describe our results in more detail. Let

$$
Z_{j}(s)=\prod_{\rho \in \mathbb{C}}(s-\rho)^{m_{j}(\rho)}
$$

be 'zeta functions' expressed as a regularized product, where

$$
m_{j}: \mathbb{C} \rightarrow \mathbb{Z}
$$

denotes the multiplicity function for $j=1, \ldots, r$. (Later, we will specify the 'zeta functions' to

Received 10 June 2002, accepted in final form 22 July 2003.

2000 Mathematics Subject Classification 11M06.

Keywords: zeta function, multiple zeta function, multiple sine function.

This journal is (C) Foundation Compositio Mathematica 2004. 


\section{Multiple Zeta FunCtions}

be treated.) We define the absolute tensor product $\left(Z_{1} \otimes \cdots \otimes Z_{r}\right)(s)$ as

$$
\left(Z_{1} \otimes \cdots \otimes Z_{r}\right)(s)=\prod_{\rho_{1}, \ldots, \rho_{r} \in \mathbb{C}}\left(s-\left(\rho_{1}+\cdots+\rho_{r}\right)\right)^{m\left(\rho_{1}, \ldots, \rho_{r}\right)}
$$

with

$$
m\left(\rho_{1}, \ldots, \rho_{r}\right)=m_{1}\left(\rho_{1}\right) \cdots m_{r}\left(\rho_{r}\right) \times \begin{cases}1 & \operatorname{Im}\left(\rho_{j}\right) \geqslant 0(j=1, \ldots, r), \\ (-1)^{r-1} & \operatorname{Im}\left(\rho_{j}\right)<0(j=1, \ldots, r), \\ 0 & \text { otherwise }\end{cases}
$$

This definition originates from [Kur92a]. We also refer to the excellent survey of Manin [Man95]. The notation of the regularized product is due to Deninger [Den92]; see [HKW03] for the required regularized products. The absolute tensor product was studied by Schröter [Sch96] under the name of 'Kurokawa tensor product'.

We are especially interested in the case of Hasse zeta functions $Z_{j}(s)=\zeta\left(s, A_{j}\right)$ for rings $A_{1}, \ldots, A_{r}$. We recall that the Hasse zeta function $\zeta(s, A)$ of a ring $A$ is defined to be

$$
\zeta(s, A)=\prod_{\mathbf{m}}\left(1-N(\mathbf{m})^{-s}\right)^{-1}
$$

where $\mathbf{m}$ runs over maximal left ideals of $A$ up to the following equivalence:

$\mathbf{m}_{1} \sim \mathbf{m}_{2} \Longleftrightarrow A / \mathbf{m}_{1}$ and $A / \mathbf{m}_{2}$ are isomorphic as left $A$-modules,

and $N(\mathbf{m})=\# \operatorname{End}_{A \text {-mod }}(A / \mathbf{m})$ (see [Kur96] and [Fuk98]). For a commutative ring $A$, the above function $\zeta(s, A)$ coincides with the usual Hasse zeta function

$$
\zeta(s, A)=\prod_{\mathbf{m}}\left(1-N(\mathbf{m})^{-s}\right)^{-1},
$$

when $\mathbf{m}$ runs over maximal ideals of $A$ and $N(\mathbf{m})=\#(A / \mathbf{m})$.

For simplicity, we write

$$
\zeta\left(s, A_{1} \otimes \cdots \otimes A_{r}\right)=\zeta\left(s, A_{1}\right) \otimes \cdots \otimes \zeta\left(s, A_{r}\right) .
$$

Actually, as was explained by Manin [Man95], we expect that our multiple zeta function would be the zeta function of the 'absolute tensor product'

$$
A_{1} \otimes_{\mathbf{F}_{1}} \cdots \otimes_{\mathbf{F}_{1}} A_{r},
$$

that is the tensor product over the (virtual) 'one element field' $\mathbf{F}_{1}$ (see [KOW03] for the absolute mathematics over ' $\mathbf{F}_{1}$ '). In any event, we note that $\zeta\left(s, A_{1} \otimes \cdots \otimes A_{r}\right)$ has the following additive structure on zeros and poles: if $\zeta\left(s, A_{j}\right)=0$ or $\infty$ and $\operatorname{Im}\left(s_{j}\right)(j=1, \ldots, r)$ all have the same sign, then $\zeta\left(s_{1}+\cdots+s_{r}, A_{1} \otimes \cdots \otimes A_{r}\right)=0$ or $\infty$.

Such an additive structure was crucial in the study of Hasse zeta functions of positive characteristic (congruence zeta functions) pursued by Grothendieck [Gro77] and Deligne [Del74], where Euler products were important for restricting the region of zeros and poles and reaching the analogue of the Riemann hypothesis.

We expect that our multiple zeta functions also have Euler products of the following form:

$$
\zeta\left(s, A_{1}\right) \otimes \cdots \otimes \zeta\left(s, A_{r}\right)=\prod_{\left(\mathbf{m}_{1}, \ldots, \mathbf{m}_{r}\right)} H_{\left(\mathbf{m}_{1}, \ldots, \mathbf{m}_{r}\right)}\left(N\left(\mathbf{m}_{1}\right)^{-s}, \ldots, N\left(\mathbf{m}_{1}\right)^{-s}\right) .
$$

Here the $\mathbf{m}_{i}$ run over the maximal (left) ideals of $A_{i}$ and $H_{\left(\mathbf{m}_{1}, \ldots, \mathbf{m}_{r}\right)}\left(T_{1}, \ldots, T_{r}\right)$ is a power series in $T_{1}, \ldots, T_{r}$ of the constant term 1 with a possible degeneration at $\left(\mathbf{m}_{1}, \ldots, \mathbf{m}_{r}\right)$ when $N\left(\mathbf{m}_{i}\right)=$ $N\left(\mathbf{m}_{j}\right)$ for some $i \neq j$. More generally, we expect that the multiple zeta function $Z_{1}(s) \otimes \cdots \otimes Z_{r}(s)$ 


\section{S. KOYAMA AND N. KUROKAWA}

has an Euler product

$$
Z_{1}(s) \otimes \cdots \otimes Z_{r}(s)=\prod_{\left(p_{1}, \ldots, p_{r}\right) \in P_{1} \times \cdots \times P_{r}} H_{\left(p_{1}, \ldots, p_{r}\right)}\left(N\left(p_{1}\right)^{-s}, \ldots, N\left(p_{r}\right)^{-s}\right)
$$

when each zeta function $Z_{j}(s)$ has an Euler product

$$
Z_{j}(s)=\prod_{p \in P_{j}} H_{p}^{j}\left(N(p)^{-s}\right)
$$

and satisfies a functional equation; here $H_{p}^{j}(T)$ is a power series in $T$ and $H_{\left(p_{1}, \ldots, p_{r}\right)}\left(T_{1}, \ldots, T_{r}\right)$ is a power series in $\left(T_{1}, \ldots, T_{r}\right)$ with a possible degeneration at $\left(p_{1}, \ldots, p_{r}\right)$ when $N\left(p_{i}\right)=N\left(p_{j}\right)$ for some $i \neq j$.

In this paper we investigate

$$
\zeta\left(s, \mathbf{F}_{p} \otimes \mathbf{F}_{q}\right)=\zeta\left(s, \mathbf{F}_{p}\right) \otimes \zeta\left(s, \mathbf{F}_{q}\right)
$$

for primes $p$ and $q$. We prove that it has a kind of Euler product expression in terms of the polylogarithm

$$
\operatorname{Li}_{k}(x):=\sum_{n=1}^{\infty} \frac{x^{n}}{n^{k}}
$$

and its variations. Note that the original 'Euler product' is

$$
\zeta\left(s, \mathbf{F}_{p}\right)=\left(1-p^{-s}\right)^{-1}=H_{p}\left(p^{-s}\right)=\exp \left(\sum_{n=1}^{\infty} \frac{p^{-n s}}{n}\right)=\exp \left(\operatorname{Li}_{1}\left(p^{-s}\right)\right),
$$

where

$$
H_{p}(T)=(1-T)^{-1}=\exp \left(\sum_{n=1}^{\infty} \frac{T^{n}}{n}\right)=\exp \left(\operatorname{Li}_{1}(T)\right) .
$$

We use the following notation for two functions $F(s)$ and $G(s): F(s) \cong G(s)$ if there exists a polynomial $Q(s)$ satisfying $F(s)=e^{Q(s)} G(s)$.

Theorem 1. For distinct primes $p$ and $q$, the following expression holds in $\operatorname{Re}(s)>0$ :

$$
\begin{aligned}
\zeta\left(s, \mathbf{F}_{p}\right) \otimes \zeta\left(s, \mathbf{F}_{q}\right) \cong & \left(1-p^{-s}\right)^{1 / 2}\left(1-q^{-s}\right)^{1 / 2} \\
& \times \exp \left(\frac{1}{2 i} \sum_{n=1}^{\infty} \frac{\cot (\pi n(\log p / \log q))}{n} p^{-n s}+\frac{1}{2 i} \sum_{n=1}^{\infty} \frac{\cot (\pi n(\log q / \log p))}{n} q^{-n s}\right) .
\end{aligned}
$$

Remark 1.1. We see that $\zeta\left(s, \mathbf{F}_{p}\right) \otimes \zeta\left(s, \mathbf{F}_{q}\right)$ is essentially $H_{p, q}\left(p^{-s}, q^{-s}\right)$ with

$$
\begin{aligned}
H_{(p, q)}\left(T_{1}, T_{2}\right)= & \left(1-T_{1}\right)^{1 / 2}\left(1-T_{2}\right)^{1 / 2} \\
& \times \exp \left(\frac{1}{2 i} \sum_{n=1}^{\infty} \frac{\cot (\pi n(\log p / \log q))}{n} T_{1}^{n}+\frac{1}{2 i} \sum_{n=1}^{\infty} \frac{\cot (\pi n(\log q / \log p))}{n} T_{2}^{n}\right),
\end{aligned}
$$

which is indeed a power series in $T_{1}$ and $T_{2}$ as we expected.

Remark 1.2. Recently, Akatsuka [Aka03] calculated $\zeta\left(s, \mathbf{F}_{p}\right) \otimes \zeta\left(s, \mathbf{F}_{q}\right) \otimes \zeta\left(s, \mathbf{F}_{r}\right)$ for distinct primes $p, q$ and $r$. For $\zeta\left(s, \mathbf{F}_{p_{1}}\right) \otimes \cdots \otimes \zeta\left(s, \mathbf{F}_{p_{r}}\right)$ see $[\mathrm{KW} 04]$.

We prove Theorem 1 by showing a corresponding expression for the double sine function. We recall the multiple sine functions studied in [Kur91, Kur92a, Kur92b, KK03a], and we use the multiple Hurwitz zeta function due to Barnes [Bar04]

$$
\zeta_{r}(s, z, \underline{\omega})=\sum_{n_{1}, \ldots, n_{r}=0}^{\infty}\left(n_{1} \omega_{1}+\cdots+n_{r} \omega_{r}+z\right)^{-s}
$$




\section{Multiple ZETA FunCtions}

for $\underline{\omega}=\left(\omega_{1}, \ldots, \omega_{r}\right)$. The definitions of the multiple gamma function and the multiple sine function are as follows:

$$
\begin{aligned}
& \Gamma_{r}(z, \underline{\omega})=\exp \left(\left.\frac{\partial}{\partial s} \zeta_{r}(s, z, \underline{\omega})\right|_{s=0}\right)=\left(\prod_{\mathbf{n} \geqslant \mathbf{0}}(\mathbf{n} \cdot \underline{\omega}+z)\right)^{-1}, \\
& S_{r}(z, \underline{\omega})=\Gamma_{r}(z, \underline{\omega})^{-1} \Gamma_{r}\left(\omega_{1}+\cdots+\omega_{r}-z, \underline{\omega}\right)^{(-1)^{r}}=\left(\prod_{\mathbf{n} \geqslant \mathbf{0}}(\mathbf{n} \cdot \underline{\omega}+z)\right)\left(\prod_{\mathbf{n} \geqslant \mathbf{1}}(\mathbf{n} \cdot \underline{\omega}-z)\right)^{(-1)^{r-1}} .
\end{aligned}
$$

When $r=2$, we have $\underline{\omega}=\left(\omega_{1}, \omega_{2}\right)$ and

$$
S_{2}\left(z,\left(\omega_{1}, \omega_{2}\right)\right)=\Gamma_{2}\left(z,\left(\omega_{1}, \omega_{2}\right)\right)^{-1} \Gamma_{2}\left(\omega_{1}+\omega_{2}-z,\left(\omega_{1}, \omega_{2}\right)\right) .
$$

We say that a real number $\alpha$ is generic if and only if

$$
\lim _{m \rightarrow \infty}\|m \alpha\|^{1 / m}=1
$$

where we put $\|x\|:=\min \{|x-n|: n \in \mathbb{Z}\}$ for $x \in \mathbb{R}$. For example:

1) if $\alpha \in(\overline{\mathbb{Q}} \cap \mathbb{R}) \backslash \mathbb{Q}$, then $\alpha$ is generic;

2) let $x, y \in \overline{\mathbb{Q}} \cap \mathbb{R}$; if $\alpha=\log x / \log y \notin \mathbb{Q}$, then $\alpha$ is transcendental and generic [Bak75, Theorem 3.1].

Theorem 2. Let $\omega_{1}$ and $\omega_{2}$ be positive real numbers. Assume that $\omega_{1} / \omega_{2}$ and $\omega_{2} / \omega_{1}$ are both generic. Then the double sine function has the following expression in $\operatorname{Im}(z)>0$ :

$$
\begin{aligned}
S_{2}\left(z,\left(\omega_{1}, \omega_{2}\right)\right)= & \exp \left(\frac{1}{2 i} \sum_{k=1}^{\infty} \frac{1}{k} \cot \left(\pi k \frac{\omega_{2}}{\omega_{1}}\right) e^{2 \pi i k\left(z / \omega_{1}\right)}+\frac{1}{2 i} \sum_{n=1}^{\infty} \frac{1}{n} \cot \left(\pi n \frac{\omega_{1}}{\omega_{2}}\right) e^{2 \pi i n\left(z / \omega_{2}\right)}\right. \\
& +\frac{1}{2} \log \left(1-e^{2 \pi i\left(z / \omega_{1}\right)}\right)+\frac{1}{2} \log \left(1-e^{2 \pi i\left(z / \omega_{2}\right)}\right) \\
& \left.+\frac{\pi i z^{2}}{2 \omega_{1} \omega_{2}}-\frac{\pi i}{2}\left(\frac{1}{\omega_{1}}+\frac{1}{\omega_{2}}\right) z+\frac{\pi i}{12}\left(\frac{\omega_{2}}{\omega_{1}}+\frac{\omega_{1}}{\omega_{2}}+3\right)\right) .
\end{aligned}
$$

Since

$$
\zeta\left(s, \mathbf{F}_{p}\right) \otimes \zeta\left(s, \mathbf{F}_{q}\right) \cong S_{2}\left(i s,\left(\frac{2 \pi}{\log p}, \frac{2 \pi}{\log q}\right)\right)
$$

by Proposition 4.1 below, Theorem 2 directly implies Theorem 1. Now the main step in proving Theorem 2 (and hence Theorem 1) is to establish the following signed double Poisson summation formula, which is a special case of signed multiple explicit formulae.

Theorem 3. Let $H(t)$ be an odd function in $L^{1}(\mathbb{R})$ with $H(t)=O\left(t^{-2}\right)$ as $|t| \rightarrow \infty$, and put

$$
\widetilde{H}(u)=\int_{-\infty}^{\infty} H(t) e^{i t u} d t .
$$

Assume that $a / b$ and $b / a$ are both generic and that the test function $H(t)$ satisfies

$$
\widetilde{H}(x)=O\left(\mu^{x}\right)
$$

as $x \rightarrow \infty$ for some $0<\mu<1$. Then we have

$$
\begin{aligned}
& \sum_{k, n>0} H\left(2 \pi\left(\frac{k}{a}+\frac{n}{b}\right)\right)+\frac{1}{2}\left(\sum_{k>0} H\left(2 \pi \frac{k}{a}\right)+\sum_{n>0} H\left(2 \pi \frac{n}{b}\right)\right) \\
& =-\frac{i a}{4 \pi} \sum_{k>0} \cot \left(\pi \frac{k a}{b}\right) \widetilde{H}(k a)-\frac{i b}{4 \pi} \sum_{n>0} \cot \left(\pi \frac{n b}{a}\right) \widetilde{H}(n b)-\frac{i a b}{8 \pi^{2}} \widetilde{H}^{\prime}(0) .
\end{aligned}
$$




\section{S. KOYAMA AND N. KUROKAWA}

We prove Theorem 3 in $\S 2$ using a method that goes back to Cramér [Cra19] (see also [Den98, Vor03]). Then we prove Theorem 2 by applying Theorem 3 in $\S 3$. We show Theorem 1 from Theorem 2 in $\S 4$. Finally, we treat $\zeta\left(s, \mathbf{F}_{p}\right) \otimes \zeta\left(s, \mathbf{F}_{p}\right)$ in $\S 5$. Our method of using the signed multiple explicit formula is applicable to general multiple zeta functions. In fact, in a forthcoming paper we will calculate the double Riemann zeta function $\zeta(s) \otimes \zeta(s)$ using this method.

\section{Signed double Poisson summation formula}

In this section we prove Theorem 3.

Lemma 2.1. Assume that $\alpha$ is generic. Then the power series

$$
\sum_{n=1}^{\infty} \cot (\pi n \alpha) x^{n}
$$

converges absolutely in $|x|<1$.

Proof. As $\alpha$ is generic, we have $\|n \alpha\|^{-1}=O\left(e^{\varepsilon n}\right)$ as $n \rightarrow \infty$ for any $\varepsilon>0$. Since $\cot (\pi x) \sim 1 /(\pi x)$ as $x \rightarrow 0$, we have $\cot (\pi n \alpha)=O\left(e^{\varepsilon n}\right)$ for any $\varepsilon>0$.

Proof of Theorem 3. We use Cramér's method from [Cra19]. Put $Z_{a}(s)=\sinh (a s / 2)$ and $Z_{b}(s)=$ $\sinh (b s / 2)$. Let $D_{T}$ be the region defined by

$$
D_{T}=\{s \in \mathbb{C}|| s|>\alpha,| \operatorname{Re}(s) \mid<\alpha, 0<\operatorname{Im}(s)<T\}
$$

with $0<\alpha<\min \{2 \pi / a, 2 \pi / b\}$. By Cauchy's theorem for an odd function $h$ which is regular in $D_{T}$, we have

$$
\sum_{\substack{0<\operatorname{Im}\left(\rho_{a}\right) \\ \operatorname{Im}\left(\rho_{b}\right)<T}} h\left(\rho_{a}+\rho_{b}\right)=\frac{1}{(2 \pi i)^{2}} \int_{\partial D_{T}} \int_{\partial D_{T}} h\left(s_{1}+s_{2}\right) \frac{Z_{a}^{\prime}}{Z_{a}}\left(s_{1}\right) \frac{Z_{b}^{\prime}}{Z_{b}}\left(s_{2}\right) d s_{1} d s_{2},
$$

where $\rho_{a}$ and $\rho_{b}$ denote the zeros of $Z_{a}(s)$ and $Z_{b}(s)$, respectively, and the integrals along $\partial D_{T}$ are taken counter-clockwise. Considering the limits as $T \rightarrow \infty$ of both sides of (2.2), we have

$$
\sum_{\operatorname{Im}\left(\rho_{a}\right), \operatorname{Im}\left(\rho_{b}\right)>0} h\left(\rho_{a}+\rho_{b}\right)=\frac{1}{(2 \pi i)^{2}} \int_{\partial D} \int_{\partial D} h\left(s_{1}+s_{2}\right) \frac{Z_{a}^{\prime}}{Z_{a}}\left(s_{1}\right) \frac{Z_{b}^{\prime}}{Z_{b}}\left(s_{2}\right) d s_{1} d s_{2},
$$

where

$$
D=\{s \in \mathbb{C}|| \operatorname{Re}(s)|<\alpha,| s \mid>\alpha, \operatorname{Im}(s)>0\} .
$$

We decompose $\partial D=C_{1} \cup C_{2} \cup C_{3}$ with

$$
C_{1}=\{s \in \partial D \mid \operatorname{Re}(s)=-\alpha\}, \quad C_{2}=\{s \in \partial D|| s \mid=\alpha\}, \quad C_{3}=\{s \in \partial D \mid \operatorname{Re}(s)=\alpha\} .
$$

We compute each double integral $I_{i j}=\left(1 /(2 \pi i)^{2}\right) \int_{C_{i}} \int_{C_{j}}$ in $(2.3)$.

First we treat the integral along the vertical lines:

$$
I_{33}=\frac{1}{(2 \pi)^{2}} \int_{0}^{\infty} \int_{0}^{\infty} h\left(2 \alpha+i\left(t_{1}+t_{2}\right)\right) \frac{Z_{a}^{\prime}}{Z_{a}}\left(\alpha+i t_{1}\right) \frac{Z_{b}^{\prime}}{Z_{b}}\left(\alpha+i t_{2}\right) d t_{1} d t_{2} .
$$

Note that

$$
\frac{Z_{a}^{\prime}}{Z_{a}}\left(\alpha+i t_{1}\right)=\frac{a}{2}+a \sum_{k=1}^{\infty} e^{-k a\left(\alpha+i t_{1}\right)}
$$




\section{Multiple ZETA FUnCTIONS}

and

$$
\frac{Z_{b}^{\prime}}{Z_{b}}\left(\alpha+i t_{2}\right)=\frac{b}{2}+b \sum_{n=1}^{\infty} e^{-n b\left(\alpha+i t_{2}\right)}
$$

We define

$$
H_{\alpha}(t)=h(2 \alpha+i t)
$$

Then (2.4) can be written as

$$
I_{33}=\frac{1}{4 \pi^{2}} \sum_{k, n \geqslant 0} \varepsilon_{k, n} a b \int_{0}^{\infty} \int_{0}^{t} H_{\alpha}(t) e^{-k a\left(\alpha+i t_{1}\right)} e^{-n b\left(\alpha+i\left(t-t_{1}\right)\right)} d t_{1} d t,
$$

where we put

$$
\varepsilon_{k, n}= \begin{cases}\frac{1}{4} & k=n=0 \\ \frac{1}{2} & k=0, n \neq 0 \text { or } k \neq 0, n=0 \\ 1 & \text { otherwise }\end{cases}
$$

Thus

$$
\begin{aligned}
I_{33}= & \frac{a b}{4 \pi^{2}} \sum_{\substack{k, n \geqslant 0 \\
k a \neq n b}} \varepsilon_{k, n} e^{-(k a+n b) \alpha} \int_{0}^{\infty} \frac{H_{\alpha}(t)\left(e^{-i k a t}-e^{-i n b t}\right)}{-i(k a-n b)} d t \\
& +\frac{a b}{4 \pi^{2}} \sum_{\substack{k, n \geqslant 0 \\
k a=n b}} \varepsilon_{k, n} e^{-2 k a \alpha} \int_{0}^{\infty} t H_{\alpha}(t) e^{-i k a t} d t .
\end{aligned}
$$

Similarly, we compute

$$
\begin{aligned}
I_{11}= & -\frac{a b}{4 \pi^{2}} \sum_{\substack{k, n \geqslant 0 \\
k a \neq n b}} \varepsilon_{k, n} e^{-(k a+n b) \alpha} \int_{0}^{\infty} \frac{H_{\alpha}(-t)\left(e^{i k a t}-e^{i n b t}\right)}{i(k a-n b)} d t \\
& -\frac{a b}{4 \pi^{2}} \sum_{\substack{k, n \geqslant 0 \\
k a=n b}} \varepsilon_{k, n} e^{-2 k a \alpha} \int_{0}^{\infty} t H_{\alpha}(-t) e^{i k a t} d t .
\end{aligned}
$$

By (2.5) and (2.6) we have

$$
\begin{aligned}
I_{11}+I_{33}= & \frac{a b}{4 \pi^{2}} \sum_{\substack{k, n \geqslant 0 \\
k a \neq n b}} \varepsilon_{k, n} e^{-(k a+n b) \alpha} \int_{-\infty}^{\infty} \frac{H_{\alpha}(t)\left(e^{-i k a t}-e^{-i n b t}\right)}{-i(k a-n b)} d t \\
& +\frac{a b}{4 \pi^{2}} \sum_{\substack{k, n \geqslant 0 \\
k a=n b}} \varepsilon_{k, n} e^{-2 k a \alpha} \int_{-\infty}^{\infty} t H_{\alpha}(t) e^{-i k a t} d t . \\
= & \frac{i a b}{4 \pi^{2}} \sum_{\substack{k, n \geqslant 0 \\
k a \neq n b}} \varepsilon_{k, n} \frac{e^{-(k a+n b) \alpha}}{k a-n b}\left(\widetilde{H_{\alpha}}(-k a)-\widetilde{H_{\alpha}}(-n b)\right) \\
& +\frac{a b}{4 \pi^{2}} \sum_{\substack{k, n \geqslant 0 \\
k a=n b}} \varepsilon_{k, n} e^{-2 k a \alpha} \widehat{t H_{\alpha}(t)}(-k a) .
\end{aligned}
$$

The assumption that $a / b$ is generic implies that the second sum consists of only one term with $k=n=0$. 


\section{S. KOYAMA AND N. KUROKAWA}

Next we calculate $I_{13}$. Since $h\left(i\left(t_{1}+t_{2}\right)\right)=H_{0}\left(t_{1}+t_{2}\right)$ and $Z_{a}^{\prime} / Z_{a}$ is an odd function, we have

$$
\begin{aligned}
I_{13} & =\frac{-1}{(2 \pi i)^{2}} \int_{\infty}^{0} \int_{0}^{\infty} h\left(i\left(t_{1}+t_{2}\right)\right) \frac{Z_{a}^{\prime}}{Z_{a}}\left(-\alpha+i t_{1}\right) \frac{Z_{b}^{\prime}}{Z_{b}}\left(\alpha+i t_{2}\right) d t_{1} d t_{2} \\
& =\frac{a b}{4 \pi^{2}} \sum_{(k, n) \neq(0,0)} \varepsilon_{k, n} e^{-(k a+n b) \alpha} \int_{0}^{\infty} \frac{H_{0}(t)\left(e^{i k a t}-e^{-i n b t}\right)}{i(k a+n b)} d t+\frac{a b}{16 \pi^{2}} \int_{0}^{\infty} t H_{0}(t) d t .
\end{aligned}
$$

Similarly,

$$
I_{31}=\frac{a b}{4 \pi^{2}} \sum_{(k, n) \neq(0,0)} \varepsilon_{k, n} e^{-(k a+n b) \alpha} \int_{0}^{\infty} \frac{H_{0}(-t)\left(e^{-i k a t}-e^{i n b t}\right)}{i(k a+n b)} d t+\frac{a b}{16 \pi^{2}} \int_{0}^{\infty} t H_{0}(t) d t .
$$

Therefore, (2.7) and (2.8) lead to

$$
I_{13}+I_{31}=-\frac{i a b}{4 \pi^{2}} \sum_{(k, n) \neq(0,0)} \varepsilon_{k, n} \frac{e^{-(k a+n b) \alpha}}{(k a+n b)}\left(\widetilde{H_{0}}(k a)-\widetilde{H_{0}}(-n b)\right)-\frac{i a b}{16 \pi^{2}} \widetilde{H}_{0}^{\prime}(0) .
$$

Letting $\alpha \rightarrow 0$ gives

$$
\lim _{\alpha \rightarrow 0}\left(I_{11}+I_{33}+I_{13}+I_{31}\right)=-\frac{i a b}{2 \pi^{2}} \sum_{(k, n) \neq(0,0)} \varepsilon_{k, n} \frac{\widetilde{H_{0}}(k a) k a-\widetilde{H}_{0}(n b) n b}{k^{2} a^{2}-n^{2} b^{2}}-\frac{i a b}{8 \pi^{2}} \widetilde{H}_{0}^{\prime}(0),
$$

since ${\widetilde{H_{0}}}^{\prime}=i \widetilde{t H_{0}(t)}$.

Next we treat $I_{2}:=I_{21}+I_{22}+I_{23}$. We compute

$$
\begin{aligned}
I_{2} & =\frac{1}{2 \pi i} \int_{C_{2}}\left(\frac{1}{2 \pi i} \int_{\partial D} h\left(s_{1}+s_{2}\right) \frac{Z_{a}^{\prime}}{Z_{a}}\left(s_{1}\right) d s_{1}\right) \frac{Z_{b}^{\prime}}{Z_{b}}\left(s_{2}\right) d s_{2} \\
& =\frac{1}{2 \pi i} \int_{C_{2}} \sum_{\rho_{a}} h\left(\rho_{a}+s_{2}\right) \frac{Z_{b}^{\prime}}{Z_{b}}\left(s_{2}\right) d s_{2},
\end{aligned}
$$

where $\rho_{a}$ runs through the zeros of $Z_{a}(s)$ with $\operatorname{Im}(\rho)>0$. Putting $s_{2}=\alpha e^{i \theta}$, we reach

$$
\lim _{\alpha \rightarrow 0} I_{2}=\frac{1}{2 \pi} \int_{\pi}^{0} \sum_{\rho_{a}} h\left(\rho_{a}\right) d \theta=-\frac{1}{2} \sum_{\rho_{a}} h\left(\rho_{a}\right) .
$$

We similarly deal with $I_{2}^{\prime}:=I_{12}+I_{22}+I_{32}$ to obtain

$$
\lim _{\alpha \rightarrow 0} I_{2}^{\prime}=-\frac{1}{2} \sum_{\rho_{b}} h\left(\rho_{b}\right)
$$

The integral $I_{22}$, which appears in both (2.10) and (2.11), tends to 0 as $\alpha \rightarrow 0$. Thus, taking (2.9), (2.10) and (2.11) into account, (2.3) equals

$$
-\frac{i a b}{2 \pi^{2}} \sum_{(k, n) \neq(0,0)} \varepsilon_{k, n} \frac{\widetilde{H}_{0}(k a) k a-\widetilde{H}_{0}(n b) n b}{k^{2} a^{2}-n^{2} b^{2}}-\frac{i a b}{8 \pi^{2}} \widetilde{H}_{0}^{\prime}(0)-\frac{1}{2} \sum_{k>0} H_{0}\left(2 \pi \frac{k}{a}\right)-\frac{1}{2} \sum_{n>0} H_{0}\left(2 \pi \frac{n}{b}\right) .
$$

Theorem 3 now follows from the formulae

$$
\sum_{n>0} \frac{2 k a}{k^{2} a^{2}-n^{2} b^{2}}+\frac{1}{k a}=\frac{\pi}{b} \cot \left(\pi \frac{k a}{b}\right), \quad \sum_{k>0} \frac{2 n b}{n^{2} b^{2}-k^{2} a^{2}}+\frac{1}{n b}=\frac{\pi}{a} \cot \left(\pi \frac{n b}{a}\right) .
$$

Remark 2.2. When we apply calculations of the above type, in the style of Cramér [Cra19], to zeta functions $Z_{1}(s)$ and $Z_{2}(s)$ that have Euler products with functional equations instead of $\sinh (a s / 2)$ and $\sinh (b s / 2)$, we obtain the signed double explicit formula. 


\section{Multiple ZETA FUnCTIONS}

\section{Expression of the double sine function}

We prove Theorem 2 from Theorem 3 .

LEMMA 3.1. We have

$$
\frac{d^{2}}{d z^{2}} \log \left(1-e^{i a z}\right)=-\sum_{n=-\infty}^{\infty} \frac{1}{(z-2 \pi n / a)^{2}}
$$

Proof. Since

$$
\log \left(1-e^{i a z}\right)=-\frac{\pi i}{2}+\frac{i a z}{2}+\log \left(2 \sin \frac{a z}{2}\right)
$$

and

$$
2 \sin \frac{a z}{2}=a z \prod_{n=1}^{\infty}\left(1-\left(\frac{a z}{2 \pi n}\right)^{2}\right)
$$

we have

$$
\frac{d^{2}}{d z^{2}} \log \left(1-e^{i a z}\right)=-\frac{1}{z^{2}}-\sum_{n=1}^{\infty}\left(\frac{1}{(z-2 \pi n / a)^{2}}+\frac{1}{(z+2 \pi n / a)^{2}}\right)=-\sum_{n=-\infty}^{\infty} \frac{1}{(z-2 \pi n / a)^{2}} .
$$

Proof of Theorem 2. Apply the odd function

$$
H(t)=\frac{1}{(z-t)^{2}}-\frac{1}{(z+t)^{2}}
$$

with $z \in \mathbb{C}, \operatorname{Im}(z)>0$, to our summation formula (1.2). As we have

$$
\widetilde{H}(x)=\int_{-\infty}^{\infty} H(t) e^{i x t} d t=2 \pi i \operatorname{Res}_{t=z}\left(H(t) e^{i x t}\right)=-2 \pi x e^{i x z},
$$

condition (1.1) is satisfied. Since $\widetilde{H}^{\prime}(0)=-2 \pi$, if we put

$$
\begin{aligned}
F(z)= & \sum_{k, n \geqslant 1}\left(\frac{1}{(z-2 \pi(k / a+n / b))^{2}}-\frac{1}{(z+2 \pi(k / a+n / b))^{2}}\right) \\
& +\frac{1}{2} \sum_{k>0}\left(\frac{1}{(z-2 \pi(k / a))^{2}}-\frac{1}{(z+2 \pi(k / a))^{2}}\right)+\frac{1}{2} \sum_{n>0}\left(\frac{1}{(z-2 \pi(n / b))^{2}}-\frac{1}{(z+2 \pi(n / b))^{2}}\right),
\end{aligned}
$$

the summation formula (1.2) gives

$$
\begin{aligned}
F(z) & =\frac{i}{2} \sum_{k>0} \cot \left(\pi \frac{k a}{b}\right) k a^{2} e^{i k a z}+\frac{i}{2} \sum_{n>0} \cot \left(\pi \frac{n b}{a}\right) n b^{2} e^{i n b z}+\frac{i a b}{4 \pi} \\
& =\frac{d^{2}}{d z^{2}}\left(\frac{1}{2 i} \sum_{k>0} \frac{1}{k} \cot \left(\pi \frac{k a}{b}\right) e^{i k a z}+\frac{1}{2 i} \sum_{n>0} \frac{1}{n} \cot \left(\pi \frac{n b}{a}\right) e^{i n b z}\right)+\frac{i a b}{4 \pi} .
\end{aligned}
$$

Now we have (see [KK03a])

$$
S_{2}\left(z, \omega_{1}, \omega_{2}\right)=e^{c_{0}+c_{1} z+c_{2} z^{2}} \frac{z \prod_{n_{1}, n_{2} \geqslant 0}^{\prime} P_{2}\left(-z /\left(n_{1} \omega_{1}+n_{2} \omega_{2}\right)\right)}{\prod_{n_{1}, n_{2} \geqslant 1} P_{2}\left(z /\left(n_{1} \omega_{1}+n_{2} \omega_{2}\right)\right)}
$$

for $P_{2}(u):=(1-u) \exp \left(u+u^{2} / 2\right)$. Hence, setting $n_{1}=k, n_{2}=n, \omega_{1}=2 \pi / a$ and $\omega_{2}=2 \pi / b$, we 


\section{S. KOYAMA AND N. KUROKAWA}

have

$$
\begin{aligned}
\frac{d^{2}}{d z^{2}} \log S_{2}\left(z,\left(\omega_{1}, \omega_{2}\right)\right)= & -\frac{1}{z^{2}}-\sum_{n_{1}, n_{2} \geqslant 1}\left(\frac{1}{\left(z+n_{1} \omega_{1}+n_{2} \omega_{2}\right)^{2}}-\frac{1}{\left(z-\left(n_{1} \omega_{1}+n_{2} \omega_{2}\right)\right)^{2}}\right) \\
& -\sum_{n_{1} \geqslant 1} \frac{1}{\left(z+n_{1} \omega_{1}\right)^{2}}-\sum_{n_{2} \geqslant 1} \frac{1}{\left(z+n_{2} \omega_{2}\right)^{2}}+2 c_{2} \\
= & F(z)-\frac{1}{2} \sum_{k=-\infty}^{\infty} \frac{1}{(z-2 \pi(k / a))^{2}}-\frac{1}{2} \sum_{n=-\infty}^{\infty} \frac{1}{(z-2 \pi(n / b))^{2}}+2 c_{2} \\
= & \frac{d^{2}}{d z^{2}}\left(\frac{1}{2 i} \sum_{k>0} \frac{1}{k} \cot \left(\pi \frac{k a}{b}\right) e^{i k a z}+\frac{1}{2 i} \sum_{n>0} \frac{1}{n} \cot \left(\pi \frac{n b}{a}\right) e^{i n b z}\right. \\
& \left.+\frac{1}{2} \log \left(1-e^{i a z}\right)+\frac{1}{2} \log \left(1-e^{i b z}\right)\right)+2 c_{2}+\frac{i a b}{4 \pi},
\end{aligned}
$$

where we have used (3.1) and Lemma 3.1. So, if we put

$$
\begin{aligned}
E(z):= & \log S_{2}\left(z,\left(\omega_{1}, \omega_{2}\right)\right)-\left(\frac{1}{2 i} \sum_{k>0} \frac{1}{k} \cot \left(\pi \frac{k \omega_{2}}{\omega_{1}}\right) e^{2 \pi i k z / \omega_{1}}\right. \\
& \left.+\frac{1}{2 i} \sum_{n>0} \frac{1}{n} \cot \left(\pi \frac{n \omega_{1}}{\omega_{2}}\right) e^{2 \pi i n z / \omega_{2}}+\frac{1}{2} \log \left(1-e^{\left(2 \pi i / \omega_{1}\right) z}\right)+\frac{1}{2} \log \left(1-e^{\left(2 \pi i / \omega_{2}\right) z}\right)\right),
\end{aligned}
$$

it follows that $d^{2} E(z) / d z^{2}$ is constant and that $E(z)$ is a polynomial of degree 2 .

Thus, we put $E(z)=\alpha+\beta z+\gamma z^{2}$ and compute $\alpha, \beta$ and $\gamma$. We first calculate $\beta$ and $\gamma$ by considering

$$
E\left(z+\omega_{1}\right)-E(z)=\left(\beta \omega_{1}+\gamma \omega_{1}^{2}\right)+2 \gamma \omega_{1} z
$$

It follows from (3.4) that (3.5) equals

$$
\begin{aligned}
\log \frac{S_{2}\left(z+\omega_{1},\left(\omega_{1}, \omega_{2}\right)\right)}{S_{2}\left(z,\left(\omega_{1}, \omega_{2}\right)\right)}-\frac{1}{2 i} \sum_{n>0} \frac{1}{n} \cot \left(\pi \frac{n \omega_{1}}{\omega_{2}}\right)\left(e^{2 \pi i n \omega_{1} / \omega_{2}}-1\right) e^{2 \pi i n z / \omega_{2}} \\
-\frac{1}{2} \log \left(1-e^{\left(2 \pi i / \omega_{2}\right)\left(z+\omega_{1}\right)}\right)+\frac{1}{2} \log \left(1-e^{\left(2 \pi i / \omega_{2}\right) z}\right) .
\end{aligned}
$$

The sum over $n$ is computed as

$$
\begin{aligned}
-\frac{1}{2 i} \sum_{n>0} \frac{1}{n} \cot \left(\pi \frac{n \omega_{1}}{\omega_{2}}\right)\left(e^{2 \pi i n \omega_{1} / \omega_{2}}-1\right) e^{2 \pi i n z / \omega_{2}} & =-\frac{1}{2} \sum_{n>0} \frac{1}{n}\left(1+e^{2 \pi i n \omega_{1} / \omega_{2}}\right) e^{2 \pi i n z / \omega_{2}} \\
& =\frac{1}{2} \log \left(1-e^{\left(2 \pi i / \omega_{2}\right)\left(z+\omega_{1}\right)}\right)+\frac{1}{2} \log \left(1-e^{\left(2 \pi i / \omega_{2}\right) z}\right) .
\end{aligned}
$$

We use formula (2.4) from [KK03a] to obtain

$$
\frac{S_{2}\left(z+\omega_{1},\left(\omega_{1}, \omega_{2}\right)\right)}{S_{2}\left(z,\left(\omega_{1}, \omega_{2}\right)\right)}=S_{1}\left(z, \omega_{2}\right)^{-1}=\left(2 \sin \frac{\pi z}{\omega_{2}}\right)^{-1} .
$$

Hence, (3.5) is equal to

$$
\begin{aligned}
-\log \left(2 \sin \frac{\pi z}{\omega_{2}}\right)+\log \left(1-e^{\left(2 \pi i / \omega_{2}\right) z}\right) & =-\log \left(2 \sin \frac{\pi z}{\omega_{2}}\right)+\log \left(-2 i e^{\left(\pi i / \omega_{2}\right) z} \sin \frac{\pi z}{\omega_{2}}\right) \\
& =-\frac{\pi i}{2}+\frac{\pi i}{\omega_{2}} z .
\end{aligned}
$$

Therefore, we have

$$
\beta \omega_{1}+\gamma \omega_{1}^{2}=-\frac{\pi i}{2} \quad \text { and } \quad 2 \gamma \omega_{1}=\frac{\pi i}{\omega_{2}}
$$




\section{Multiple Zeta FunCtions}

We thus obtain

$$
\beta=-\frac{\pi i}{2}\left(\frac{1}{\omega_{1}}+\frac{1}{\omega_{2}}\right) \quad \text { and } \quad \gamma=\frac{\pi i}{2 \omega_{1} \omega_{2}} .
$$

Next we deal with $\alpha$ by considering

$$
E(z)+E\left(z+\frac{\omega_{1}}{2}\right)+E\left(z+\frac{\omega_{2}}{2}\right)+E\left(z+\frac{\omega_{1}+\omega_{2}}{2}\right)-E(2 z) .
$$

The constant term of $(3.6)$ is

$$
3 \alpha+\beta\left(\omega_{1}+\omega_{2}\right)+\gamma\left(\frac{\omega_{1}}{4}+\frac{\omega_{2}}{4}+\frac{\left(\omega_{1}+\omega_{2}\right)^{2}}{4}\right)=3 \alpha-\frac{\pi i}{4}\left(\frac{\omega_{2}}{\omega_{1}}+\frac{\omega_{1}}{\omega_{2}}+3\right) .
$$

On the other hand, we compute (3.6) by using (3.4). We write (3.6) as $\sum_{j=0}^{4} A_{j}$, where

$$
\begin{aligned}
& A_{0}=\log \frac{S_{2}\left(z,\left(\omega_{1}, \omega_{2}\right)\right) S_{2}\left(z+\omega_{1} / 2,\left(\omega_{1}, \omega_{2}\right)\right) S_{2}\left(z+\omega_{2} / 2,\left(\omega_{1}, \omega_{2}\right)\right) S_{2}\left(z+\left(\omega_{1}+\omega_{2}\right) / 2,\left(\omega_{1}, \omega_{2}\right)\right)}{S_{2}\left(2 z,\left(\omega_{1}, \omega_{2}\right)\right)}, \\
& A_{1}=-\frac{1}{2 i} \sum_{k>0} \frac{\cot \left(\pi\left(k \omega_{2} / \omega_{1}\right)\right)}{k}\left(e^{\frac{2 \pi i k z}{\omega_{1}}}+e^{\frac{2 \pi i k}{\omega_{1}}\left(z+\frac{\omega_{1}}{2}\right)}+e^{\frac{2 \pi i k}{\omega_{1}}\left(z+\frac{\omega_{2}}{2}\right)}+e^{\frac{2 \pi i k}{\omega_{1}}\left(z+\frac{\omega_{1}+\omega_{2}}{2}\right)}-e^{\frac{4 \pi i k z}{\omega_{1}}}\right) \text {, } \\
& A_{2}=-\frac{1}{2 i} \sum_{n>0} \frac{\cot \left(\pi\left(n \omega_{1} / \omega_{2}\right)\right)}{n}\left(e^{\frac{2 \pi i n z}{\omega_{2}}}+e^{\frac{2 \pi i n}{\omega_{2}}\left(z+\frac{\omega_{2}}{2}\right)}+e^{\frac{2 \pi i n}{\omega_{2}}\left(z+\frac{\omega_{1}}{2}\right)}+e^{\frac{2 \pi i n}{\omega_{2}}\left(z+\frac{\omega_{2}+\omega_{1}}{2}\right)}-e^{\frac{4 \pi i n z}{\omega_{2}}}\right) \text {, } \\
& A_{3}=-\frac{1}{2} \log \frac{\left(1-e^{\frac{2 \pi i}{\omega_{1}} z}\right)\left(1-e^{\frac{2 \pi i}{\omega_{1}}\left(z+\frac{\omega_{1}}{2}\right)}\right)\left(1-e^{\frac{2 \pi i}{\omega_{1}}\left(z+\frac{\omega_{2}}{2}\right)}\right)\left(1-e^{\frac{2 \pi i}{\omega_{1}}\left(z+\frac{\omega_{1}+\omega_{2}}{2}\right)}\right)}{1-e^{\frac{4 \pi i}{\omega_{1}} z}}, \\
& A_{4}=-\frac{1}{2} \log \frac{\left(1-e^{\frac{2 \pi i}{\omega_{2}} z}\right)\left(1-e^{\frac{2 \pi i}{\omega_{2}}\left(z+\frac{\omega_{1}}{2}\right)}\right)\left(1-e^{\frac{2 \pi i}{\omega_{2}}\left(z+\frac{\omega_{2}}{2}\right)}\right)\left(1-e^{\frac{2 \pi i}{\omega_{2}}\left(z+\frac{\omega_{1}+\omega_{2}}{2}\right)}\right)}{1-e^{\frac{4 \pi i}{\omega_{2}} z}} .
\end{aligned}
$$

Formula (2.5) from [KK03a] gives $A_{0}=0$. Next, $A_{1}$ is computed as follows:

$$
\begin{aligned}
A_{1} & =-\frac{1}{2 i} \sum_{\substack{k>0 \\
\text { even }}} \frac{1}{k} \cot \left(\pi \frac{k \omega_{2}}{\omega_{1}}\right)\left(2 e^{\frac{2 \pi i k z}{\omega_{1}}}+2 e^{\frac{2 \pi i k}{\omega_{1}}\left(z+\frac{\omega_{2}}{2}\right)}\right)+\frac{1}{2 i} \sum_{k>0} \frac{1}{k} \cot \left(\pi \frac{k \omega_{2}}{\omega_{1}}\right) e^{\frac{4 \pi i k z}{\omega_{1}}} \\
& =-\frac{1}{2 i} \sum_{k>0} \frac{1}{k}\left(\cot \left(\pi \frac{2 k \omega_{2}}{\omega_{1}}\right)\left(1+e^{\frac{2 \pi i k \omega_{2}}{\omega_{1}}}\right)-\cot \left(\pi \frac{k \omega_{2}}{\omega_{1}}\right)\right) e^{\frac{4 \pi i k z}{\omega_{1}}} \\
& =-\frac{1}{2} \sum_{k>0} \frac{1}{k} e^{\frac{2 \pi i k \omega_{2}}{\omega_{1}}} e^{\frac{4 \pi i k z}{\omega_{1}}} \\
& =\frac{1}{2} \log \left(1-e^{\frac{4 \pi i}{\omega_{1}}\left(z+\frac{\omega_{2}}{2}\right)}\right) .
\end{aligned}
$$

Here we have used the identity

$$
\cot 2 \theta\left(1+e^{2 i \theta}\right)-\cot \theta=i e^{2 i \theta}
$$

with $\theta=\pi\left(k \omega_{2} / \omega_{1}\right)$. Similarly, $A_{2}$ is calculated as

$$
A_{2}=\frac{1}{2} \log \left(1-e^{\frac{4 \pi i}{\omega_{2}}\left(z+\frac{\omega_{1}}{2}\right)}\right) .
$$

The remaining terms are easily computed as

$$
A_{3}=-\frac{1}{2} \log \left(1-e^{\frac{4 \pi i}{\omega_{1}}\left(z+\frac{\omega_{2}}{2}\right)}\right), \quad A_{4}=-\frac{1}{2} \log \left(1-e^{\frac{4 \pi i}{\omega_{2}}\left(z+\frac{\omega_{1}}{2}\right)}\right) .
$$




\section{S. Koyama AND N. Kurokawa}

Hence we have deduced that (3.6) is equal to $\sum_{j=0}^{4} A_{j}=0$. Therefore, its constant term (3.7) vanishes, which leads to

$$
\alpha=\frac{\pi i}{12}\left(\frac{\omega_{1}}{\omega_{2}}+\frac{\omega_{2}}{\omega_{1}}+3\right) .
$$

Remark 3.2. The referee indicated that the method used above to prove Theorem 2 may be modified as follows. Let $R(z)$ be the quotient of the left-hand side by the right-hand side in Theorem 2 . Using the formulae

$$
\frac{S_{2}\left(z,\left(\omega_{1}, \omega_{2}\right)\right)}{S_{2}\left(z+\omega_{1},\left(\omega_{1}, \omega_{2}\right)\right)}=2 \sin \frac{\pi z}{\omega_{2}}, \quad \frac{S_{2}\left(z,\left(\omega_{1}, \omega_{2}\right)\right)}{S_{2}\left(z+\omega_{2},\left(\omega_{1}, \omega_{2}\right)\right)}=2 \sin \frac{\pi z}{\omega_{1}}
$$

and exponential expressions for trigonometric functions, we see that $R(z)$ is both $\omega_{1}$-periodic and $\omega_{2}$-periodic. Hence $R(z)$ is constant, since $\omega_{1} / \omega_{2}$ is an irrational real number. To calculate the constant function $R(z)$ one uses the natural branch of $\log R(z)$ and evaluates

$$
3 \log R(z)=\sum_{\omega \in T} \log R(z+\omega)-\log R(2 z),
$$

where $T=\left\{0, \omega_{1} / 2, \omega_{2} / 2,\left(\omega_{1}+\omega_{2}\right) / 2\right\}$. For this, one applies the following functional equation for the natural branch of $\log S_{2}\left(z,\left(\omega_{1}, \omega_{2}\right)\right)$ in the strip $0<\operatorname{Re}(z)<\omega_{1}+\omega_{2}$ :

$$
\sum_{\omega \in T} \log S_{2}\left(z+\omega,\left(\omega_{1}, \omega_{2}\right)\right)=\log S_{2}\left(2 z,\left(\omega_{1}, \omega_{2}\right)\right),
$$

which is immediate from the corresponding functional equation for the double Hurwitz zeta function.

Remark 3.3. The condition that $\omega_{2} / \omega_{1}$ and $\omega_{1} / \omega_{2}$ are generic is similar to the 'diophantine condition' used by Connes [Con85] to calculate cohomologies for (irrational rotational) non-commutative tori. The problem belongs to the category of so-called 'small denominator problems'.

Remark 3.4. We easily see that

$$
\frac{\pi i z^{2}}{2 \omega_{1} \omega_{2}}-\frac{\pi i}{2}\left(\frac{1}{\omega_{1}}+\frac{1}{\omega_{2}}\right) z+\frac{\pi i}{12}\left(\frac{\omega_{2}}{\omega_{1}}+\frac{\omega_{1}}{\omega_{2}}+3\right)=\pi i \zeta_{2}\left(0, z,\left(\omega_{1}, \omega_{2}\right)\right),
$$

which is a multiple Bernoulli polynomial of Barnes [Bar04].

Remark 3.5. The formula of Theorem 2 is valid for more general situations. For example, it holds for $\arg \left(\omega_{2}\right)<\arg (z)<\arg \left(\omega_{1}\right)+\pi$ when $0 \leqslant \arg \left(\omega_{1}\right) \leqslant \arg \left(\omega_{2}\right)<\pi$. We refer to [KW04] where $S_{r}\left(z ;\left(\omega_{1}, \ldots, \omega_{r}\right)\right)$ is also treated.

\section{The product $\zeta\left(s, \mathbf{F}_{p}\right) \otimes \zeta\left(s, \mathbf{F}_{q}\right)$ for distinct primes $p$ and $q$}

We first describe a convenient form of the absolute tensor product of some zeta functions. Let $Z_{j}$ $(j=1,2)$ be meromorphic functions of order $\mu_{j}$. We put the Hadamard product as

$$
Z_{j}(s)=s^{k_{j}} e^{Q_{j}(s)} \prod_{\rho \in \mathbb{C}}^{\prime} P_{\mu_{j}}\left(\frac{s}{\rho}\right)^{m_{j}(\rho)},
$$

where $P_{r}(u):=(1-u) \exp \left(u+u^{2} / 2+\cdots+u^{r} / r\right), m_{j}$ denotes the multiplicity function with $k_{j}:=m_{j}(0)$, and $Q_{j}$ is a polynomial with $\operatorname{deg} Q_{j} \leqslant \mu_{j}$. Here the product over $\rho \in \mathbb{C}$ means

$$
\lim _{R \rightarrow \infty} \prod_{0<|\rho|<R} P_{\mu_{j}}\left(\frac{s}{\rho}\right)^{m_{j}(\rho)} .
$$

Assume, moreover, that $Z_{j}$ is one of the following: the Riemann zeta function, the Hasse zeta function of a finite field, the Selberg zeta function of a circle and the Selberg zeta function of 


\section{Multiple ZETA FUnCTIONS}

a Riemann surface. Then, by [HKW03] and [KKSW03], the absolute tensor product originally constructed as a regularized product turns out to be the following Hadamard product:

$$
\left(Z_{1} \otimes Z_{2}\right)(s)=s^{k_{1} k_{2}} e^{Q(s)} \prod_{\rho_{1}, \rho_{2} \in \mathbb{C}}^{\prime} P_{\mu_{1}+\mu_{2}}\left(\frac{s}{\rho_{1}+\rho_{2}}\right)^{m\left(\rho_{1}, \rho_{2}\right)},
$$

where $Q(s)$ is a polynomial with $\operatorname{deg} Q \leqslant \mu_{1}+\mu_{2}$ and

$$
m\left(\rho_{1}, \rho_{2}\right):=m_{1}\left(\rho_{1}\right) m_{2}\left(\rho_{2}\right) \times \begin{cases}1 & \text { if } \operatorname{Im}\left(\rho_{1}\right), \operatorname{Im}\left(\rho_{2}\right) \geqslant 0, \\ -1 & \text { if } \operatorname{Im}\left(\rho_{1}\right), \operatorname{Im}\left(\rho_{2}\right)<0, \\ 0 & \text { otherwise }\end{cases}
$$

See [HKW03] regarding the existence of the (re-)regularized product for multiple zeta functions and [KKSW03] for its relation to the Hadamard product. Our restriction to four specific zeta functions can be weakened considerably by the method of [HKW03]. It is worthwhile consulting [Ill01, Ill02] for a study of regularized products.

Here we compute this absolute tensor product for Hasse zeta functions of finite fields,

$$
Z_{1}(s)=\zeta\left(s, \mathbf{F}_{p}\right)=\left(1-p^{-s}\right)^{-1}, \quad Z_{2}(s)=\zeta\left(s, \mathbf{F}_{q}\right)=\left(1-q^{-s}\right)^{-1},
$$

with $p, q$ primes.

Proposition 4.1. The absolute tensor product of Hasse zeta functions for finite prime fields is given as

$$
\zeta\left(s, \mathbf{F}_{p}\right) \otimes \zeta\left(s, \mathbf{F}_{q}\right) \cong S_{2}\left(i s,\left(\frac{2 \pi}{\log p}, \frac{2 \pi}{\log q}\right)\right)
$$

Proof. We easily compute the Hadamard product (4.1) for the Hasse zeta function, which is given by

$$
\zeta\left(s, \mathbf{F}_{p}\right) \cong s^{-1} \prod_{n=-\infty}^{\infty} P_{1}\left(\frac{s}{(2 \pi i / \log p) n}\right)^{-1}
$$

Thus, by (4.2),

$$
\zeta\left(s, \mathbf{F}_{p}\right) \otimes \zeta\left(s, \mathbf{F}_{q}\right) \cong s \prod_{k, n \in \mathbb{Z}}^{\prime} P_{2}\left(\frac{s}{(2 \pi i / \log p) k+(2 \pi i / \log q) n}\right)^{m_{k, n}}
$$

with

$$
m_{k, n}:=m\left(\frac{2 \pi i}{\log p} k, \frac{2 \pi i}{\log q} n\right)= \begin{cases}1 & \text { if } k, n \geqslant 0 \\ -1 & \text { if } k, n<0 \\ 0 & \text { otherwise }\end{cases}
$$

Hence

$$
\zeta\left(s, \mathbf{F}_{p}\right) \otimes \zeta\left(s, \mathbf{F}_{q}\right) \cong s \frac{\prod_{k, n=0}^{\infty} P_{2}\left(\frac{s}{(2 \pi i / \log p) k+(2 \pi i / \log q) n}\right)}{\prod_{k, n=1}^{\infty} P_{2}\left(-\frac{s}{(2 \pi i / \log p) k+(2 \pi i / \log q) n}\right)}
$$




\section{S. KOYAMA AND N. KUROKAWA}

We use the $r=2$ case of the formula of Proposition 2.4 from [KK03a]:

$$
S_{2}\left(z,\left(\omega_{1}, \omega_{2}\right)\right) \cong z \frac{\prod_{k, n=0}^{\infty} P_{2}\left(-z /\left(\omega_{1} k+\omega_{2} n\right)\right)}{\prod_{k, n=1}^{\infty} P_{2}\left(z /\left(\omega_{1} k+\omega_{2} n\right)\right)} .
$$

Putting $z=i s$ and $\underline{\omega}=\left(\omega_{1}, \omega_{2}\right)=(2 \pi / \log p, 2 \pi / \log q)$, we reach the proposition.

Proof of Theorem 1. We take $\left(\omega_{1}, \omega_{2}\right)=(2 \pi / \log p, 2 \pi / \log q)$ in Theorem 2. As remarked in $\S 1$, $\omega_{1} / \omega_{2}=(\log q) /(\log p)$ is generic, since $p$ and $q$ are distinct primes. Thus Proposition 4.1 gives the assertion of Theorem 1 .

Remark 4.2. By the same proof, Theorem 1 is also valid when $p$ and $q$ are powers of distinct primes.

\section{The product $\zeta\left(s, \mathbf{F}_{p}\right) \otimes \zeta\left(s, \mathbf{F}_{p}\right)$}

In this section we treat $\zeta\left(s, \mathbf{F}_{p}\right) \otimes \zeta\left(s, \mathbf{F}_{p}\right)$ for a prime number $p$.

Theorem 4. Let $p$ be a prime number. Then for $\operatorname{Re}(s)>0$ we have

$$
\zeta\left(s, \mathbf{F}_{p}\right) \otimes \zeta\left(s, \mathbf{F}_{p}\right) \cong\left(1-p^{-s}\right)^{1-i s \log p / 2 \pi} \exp \left(-\frac{\operatorname{Li}_{2}\left(p^{-s}\right)}{2 \pi i}\right) .
$$

We prove Theorem 4 from the following result.

Theorem 5. Let $\omega>0$. Then for $\operatorname{Im}(z)>0$ we have

$$
S_{2}(z,(\omega, \omega))=\exp \left(-\frac{1}{2 \pi i} \operatorname{Li}_{2}\left(e^{2 \pi i z / \omega}\right)+\pi i\left(\frac{z^{2}}{2 \omega^{2}}-\frac{z}{\omega}+\frac{5}{12}\right)\right)\left(1-e^{2 \pi i z / \omega}\right)^{1-z / \omega} .
$$

Proof. We recall the formulae of the double sine function:

$$
\begin{aligned}
S_{2}(z,(\omega, \omega)) & =S_{2}\left(\frac{z}{\omega},(1,1)\right) & & \text { [KK03a, Theorem 2.1(c) }] \\
& =\mathcal{S}_{2}\left(\frac{z}{\omega}\right)^{-1} \mathcal{S}_{1}\left(\frac{z}{\omega}\right) & & {[\text { KK03a, Example 3.6]. }}
\end{aligned}
$$

Here $\mathcal{S}_{r}(z)(r=1,2)$ are the primitive multiple sine functions [KK03a]. We have by definition

$$
\mathcal{S}_{1}(z)=2 \sin \pi z
$$

and the expression [KK03a, Theorem 2.8, (2.12)]

$$
\mathcal{S}_{2}(z)=\exp \left(\frac{1}{2 \pi i} \operatorname{Li}_{2}\left(e^{2 \pi i z}\right)+z \log \left(1-e^{2 \pi i z}\right)-\frac{\pi i}{2} z^{2}+\frac{\pi i}{12}\right)
$$

for $\operatorname{Im}(z)>0$. Then an easy calculation gives Theorem 5 .

Remark 5.1. As in Remark 3.4, we see that

$$
\pi i\left(\frac{z^{2}}{2 \omega^{2}}-\frac{z}{\omega}+\frac{5}{12}\right)=\pi i \zeta_{2}(0, z,(\omega, \omega))
$$

is again a multiple Bernoulli polynomial.

Proof of Theorem 4. Let $z=i s$ and $\omega=2 \pi / \log p$ in Theorem 5 . 


\section{Multiple ZETA FunCtions}

Remark 5.2. Theorems 4 and 5 can alternatively be proved in the same manner as Theorems 1 and 2. The Euler product (5.1) is deeply related to the generalized Kummer's formula for the double gamma function obtained in [KK03c].

Remark 5.3. For the case of powers of the same prime $p$, the calculation of the tensor product $\zeta\left(s, \mathbf{F}_{p^{k}}\right) \otimes \zeta\left(s, \mathbf{F}_{p^{l}}\right)$, a generalization of Theorem 4, is described in [KK03b].

\section{ACKNOWLEDGEMEnTs}

We thank Professor Christopher Deninger for his interest in our multiple zeta functions and for supplying the book [Sch96]. We thank the referee for making comments, including Remark 3.2.

\section{REFERENCES}

Aka03 H. Akatsuka, Euler product expression of triple zeta functions, Preprint (2003).

Bak75 A. Baker, Transcendental number theory (Cambridge University Press, 1975).

Bar04 E. W. Barnes, On the theory of the multiple gamma function, Trans. Cambridge Philos. Soc. 19 (1904), 374-425.

Con85 A. Connes, Noncommutative differential geometry, Publ. Math. Inst. Hautes Études Sci. 62 (1985), $257-360$.

Cra19 H. Cramér, Studien über die Nullstellen der Riemannschen Zetafunktion, Math. Z. 4 (1919), 104-130.

Del74 P. Deligne, La conjecture de Weil. I, Publ. Math. Inst. Hautes Études Sci. 43 (1974), 273-307.

Den92 C. Deninger, Local L-factors of motives and regularized determinants, Invent. Math. 107 (1992), $135-150$.

Den98 C. Deninger, Some analogues between number theory and dynamical systems on foliated spaces, in Proceedings of the International Congress of Mathematicians (Berlin 1998), vol. I, Doc. Math. extra vol. I (1998), 163-186.

Fuk98 T. Fukaya, Hasse zeta functions of non-commutative rings, J. Algebra 208 (1998), 304-342.

Gro77 A. Grothendieck, Séminaire de géométrie algébrique du Bois-Marie 1965-66 (SGA 5), Lecture Notes in Mathematics, vol. 589 (Springer, Berlin, 1977).

HKW03 M. Hirano, N. Kurokawa and M. Wakayama, Half zeta functions, J. Ramanujan Math. Soc. 18 (2003), 195-209.

Ill01 G. Illies, Regularized products and determinants, Comm. Math. Phys. 220 (2001), 69-94.

Ill02 G. Illies, Cramér functions and Guinand equations, Acta Arith. 105 (2002), 103-118.

KKSW03 K. Kimoto, N. Kurokawa, C. Sonoki and M. Wakayama, Zeta regularizations and q-analogue of ring sine functions, Kyushu J. Math. 57 (2003), 197-215.

Kur91 N. Kurokawa, Multiple sine functions and Selberg zeta functions, Proc. Japan Acad. Ser. A Math. Sci. 67 (1991), 61-64.

Kur92a N. Kurokawa, Multiple zeta functions: an example, Adv. Stud. Pure Math. 21 (1992), 219-226.

Kur92b N. Kurokawa, Gamma factors and Plancherel measures, Proc. Japan Acad. Ser. A Math. Sci. 68 (1992), 256-260.

Kur96 N. Kurokawa, Zeta functions of categories, Proc. Japan Acad. Ser. A Math. Sci. 72 (1996), 221-222.

KK03a N. Kurokawa and S. Koyama, Multiple sine functions, Forum Math. 15 (2003), 839-876.

KK03b N. Kurokawa and S. Koyama, Normalized double sine functions, Proc. Japan Acad. Ser. A Math. Sci. 79 (2003), 14-18.

KK03c S. Koyama and N. Kurokawa, Kummer's formula for multiple gamma functions, J. Ramanujan Math. Soc. 18 (2003), 87-107. 


\section{Multiple ZETA FunCTiOnS}

KOW03 N. Kurokawa, H. Ochiai and M. Wakayama, Absolute derivations and zeta functions, Doc. Math. Kato extra volume (2003), 565-584.

KW04 N. Kurokawa and M. Wakayama, Absolute tensor products, Int. Math. Res. Not. 2004, no. 5 (2004), 249-260.

Man95 Yu. I. Manin, Lectures on zeta functions and motives (according to Deninger and Kurokawa), Astérisque 228 (1995), 121-163.

Sch96 M. Schröter, Über Kurokawa-Tensorprodukte von L-Reihen, Schriftenreihe des Mathematischen Instituts der Universität Münster, 3. Serie, Heft 18 (1996).

Vor03 A. Voros, Zeta functions for the Riemann zeros, Ann. Inst. Fourier (Grenoble) 53 (2003), 665-699.

Shin-ya Koyama koyama@math.keio.ac.jp

Department of Mathematics, Keio University, 223-8522, Japan

Nobushige Kurokawa kurokawa@math.titech.ac.jp

Department of Mathematics, Tokyo Institute of Technology, 152-8551, Japan 